\title{
Asset management, High Water Mark and flow of funds
}

\author{
Emilio Barucci ${ }^{\mathrm{a}}$, Daniele Marazzina ${ }^{\mathrm{a}, *}$ \\ ${ }^{a}$ Politecnico di Milano, Dipartimento di Matematica, I-20133 Milano, Italy
}

\begin{abstract}
We analyze the asset manager's portfolio problem when he is remunerated through a High Water Mark incentive fee and a management fee, and the assets under management are characterized by in/outflow of funds as a function of the performance of the fund with respect to a benchmark. Once we solve numerically the investment problem, we show that the presence of a flow fund induces risk in excess in case of a High Water Mark defined on the pure performance of the fund. Instead a High Water Mark defined on the assets under management leads to a more prudent investment strategy.
\end{abstract}

Keywords: asset management, management fee, High Water Mark, flow of funds.

\section{Introduction}

Since the pioneering contribution of Merton on the intertemporal optimal investment problem [10], the literature on portfolio choices has grown up significantly. In this paper we analyze the asset manager's portfolio problem when he is remunerated through a High Water Mark (HWM) incentive fee and a management fee and the assets under management (AUM) are characterized by in/outflow of funds as a function of the performance of the fund with respect to a benchmark. We study the impact of the flow of funds on the investment strategy concentrating our attention on excess risk taking with respect to the benchmark and to the investment strategy without flow of funds.

Analyzing this problem, we contribute to two fields of research: the intertemporal portfolio problem with a nonlinear remuneration scheme, and the analysis of the effect of the in/outflow of funds on the asset manager's investment strategy.

We consider two different types of HWM remuneration schemes: a contract where the HWM is defined on the performance of the fund depurated by the in/outflow of funds, as it is in the habit of the hedge fund industry, and a contract where the HWM is defined on the AUM including the flow of funds. Once we solve numerically the optimal investment problems, we show that the first type of contract induces the manager to take risk in excess with respect to the second one, to the benchmark and to the optimal strategy without flow of funds for a large set of parameters. Instead, a HWM remuneration scheme defined on the AUM induces the manager to take an investment strategy that is intermediate between the benchmark and

\footnotetext{
* Corresponding author

Email addresses: emilio.barucci@polimi.it (Emilio Barucci), daniele.marazzina@polimi.it (Daniele Marazzina)
}

the optimal strategy without flow of funds. Also the composition of the remuneration scheme influences the manager's portfolio choice: the risk attitude of the manager is positively affected by the HWM fee and negatively affected by the management fee.

The asset manager's investment problem significantly differs from the one of the private investor. As a matter of fact, the asset manager's objective function is not related to its wealth but to the AUM and to the performance of the fund. In most of the cases, the remuneration of the manager is made up of two components: a management fee, which is proportional to the AUM, and an incentive fee which is nonlinear in the performance of the fund. These features of the remuneration scheme of the manager significantly affect the investment strategy, see [3, 4, 8]. The practice in the hedge fund industry is to consider a management fee and a HWM incentive fee, see $[6,9,11]$. These papers show that the optimal investment strategy depends upon the termination clause of the fund/outside option for the manager. Assuming that the fund is liquidated only in case the AUM reach the zero level or by an exogenous event, [11] shows that the investment strategy is of a constant weight type with a significant risk exposure; considering a liquidation triggered by a threshold on the fraction of the AUM over the HWM, the manager tends to reduce the risk exposure which turns out to be increasing in the ratio $\mathrm{AUM} / \mathrm{HWM}$, see $[6,9]$. In this context, the risk neutral asset manager defines the investment strategy balancing the risk of an early termination in case of a poor performance (downside liquidation risk) with the benefit from the continuation provided by the management and the incentive fees.

The literature on asset management and flow funds is rather limited. We refer to [2], where authors assume that the asset manager maximizes the expected utility associated with a management fee related to the AUM by the end of a finite horizon. In/outflow of funds is modeled assum- 
ing that the AUM by the end of the horizon are multiplied by a parameter that is positively and nonlinearly affected by the performance of the fund with respect to the benchmark. They obtain results similar to those obtained with a nonlinear remuneration scheme showing risk shifting when the performance of the fund runs behind the benchmark, but the exposure does not become unbounded when the performance deteriorates significantly as in the case of an option like remuneration scheme [4].

The effect of the in/outflow of funds on the portfolio problem of an asset manager remunerated through a HWM contract has not been fully analyzed yet, for the empirical evidence see $[5,12]$. $[6,9]$ consider a constant rate of outflow: [6] shows that the investment in the risky asset is increasing in the withdrawal rate, the rationale of this result is that a higher withdrawal rate reduces the revenues from the management fee leading to a higher risk appetite; [9] also assumes that there are fund inflows whenever the AUM exceed its HWM. This inflow leads to an increase of the investment in the risky asset, the rationale of this result is that the inflow in case of a good performance increases the AUM size providing an option like remuneration (via the management fee) which induces a higher risk exposure.

Note that $[6,9]$ do not model the in/outflow of funds through the relative performance of the fund with respect to a benchmark. This represents a strong limit of their analysis: as a matter of fact, although the incentive fees for hedge funds are defined in terms of absolute performance, the flow of funds is related to the relative performance of the fund with respect to its peers or to a benchmark. In this paper we address this point modeling the in/outflow of funds in continuous time as a linear function of the instantaneous performance of the fund compared to that of an exogenous benchmark. We confirm several results already obtained in $[6,9]$ and we provide some new insights. Overall we show that the manager's risk attitude in case of a HWM fee defined on the performance of the fund is higher than in case of a HWM fee defined on the AUM. The first type of contract leads to excess risk taking with respect to the benchmark and to the optimal strategy without flow of funds for a large set of parameters. The latter type of contract leads to an investment strategy that is intermediate between the optimal strategy without flow of funds and the benchmark. Analyzing the optimal investment strategy with respect to the composition of the remuneration scheme, we observe that the risk attitude of the manager is positively affected by the incentive fee and negatively affected by the management fee. All these results can be rationalized according to the trade-off between the liquidation risk and the continuation benefit.

These results show the potential negative effect that comes from a combination between a non well designed HWM contract and flow of funds. As a matter of fact, we may consider that the investor looks for an aggressive strategy by a manager of a hedge fund and therefore we may consider as "expected" the risk taking level obtained without flow of funds, instead the extra component that comes from the flow of funds is not welcomed by investors subscribing the fund and therefore in "unwanted".

The paper is organized as follows. In Section 2 we present our model, the HWM contracts and the optimization problem. In Section 3 we state the Hamilton-JacobiBellman (HJB) equation related to the value function. Finally, in Section 4 we analyze the optimal investment strategies.

\section{The model}

We consider a continuous time economy where agents trade a risk-free asset and a risky asset. The prices of the two assets evolve as follows: $d B(t)=r B(t) d t, B(0)=$ $B_{0}$, and $d S(t)=\mu S(t) d t+\sigma S(t) d Z(t), S(0)=S_{0}$, where $\mu, r$ and $\sigma$ are positive constants such that $\mu>r$ and $Z(t)$ is a one-dimensional Brownian motion on a complete probability space $(\Omega, \mathcal{F}, P)$. We denote by $\mathcal{F}=\mathcal{F}_{t}$ the $P$-augmentation of the filtration generated by $Z(t)$.

As in $[6,9,11]$, we assume that the asset manager is riskneutral. We distinguish the evolution of the AUM $(W(t))$ from the evolution of the fund on a solo basis $(F(t))$. The difference is provided by the in/outflow of funds that depends on the relative performance of the fund with respect to an exogenous benchmark $Y(t)$ (a management style benchmark for a hedge fund). In the rest of the paper we refer to $F$ as fund performance and to $W$ as AUM. We assume that $W(0)=F(0)=Y(0)$. The benchmark evolves as follows

$$
d Y(t)=Y(t)[(1-\beta) r+\beta \mu] d t+Y(t) \sigma \beta d Z(t)
$$

where $\beta$ is the benchmark (constant) weight.

Let $\theta(t)$ be the fraction of funds invested in the risky asset at time $t$. The fund performance evolves as

$$
d F(t)=F(t)[(1-\theta(t)) r+\theta(t) \mu] d t+F(t) \sigma \theta(t) d Z(t) .
$$

To have a well-defined problem, we require the usual assumption $\int_{0}^{T}(\theta(t) F(t))^{2} d t<+\infty$ for any $T>0$. $W(t)$ depends on the in/outflow of funds which is described by a linear function of the instantaneous relative performance of the fund with respect to the benchmark

$$
\begin{aligned}
& d W(t)=W(t)[(1-\theta(t)) r+\theta(t) \mu] d t+W(t) \sigma \theta(t) d Z(t) \\
& +W(t) \eta\left(\frac{d F(t)}{F(t)}-\frac{d Y(t)}{Y(t)}\right)-(\cdots),
\end{aligned}
$$

where $\eta$ provides the intensity of the flow-fund and the missing term depends on the definition of the remuneration scheme.

The manager's remuneration scheme is composed by a management fee (a constant fraction of AUM) and a HWM incentive fee. In what follows we consider two different types of HWM contracts: the first one comes from the practice in the asset management industry and defines the 
remuneration as a function of the maximum of the performance of the fund depurated by the in/outflow of funds, i.e. $F(t)$; the second one defines the remuneration with respect to the maximum of the AUM, i.e. $W(t)$, including performance and in/outflow of funds. In the following, we refer to these contracts as the pure performance running maximum $(\mathrm{P})$ and the assets under management running maximum (AUM), respectively.

Let us consider first the pure performance running maximum HWM contract. HWM remuneration schemes are defined with respect to the maximum of the performance of the fund $(F(t))$. Introducing that state variable renders the analysis quite complicate: to maintain analytical and numerical tractability, we follow $[6,9]$ and we design the HWM as the maximum of the AUM $W(t)$ corrected for the flow funds. To this end, we denote our reference variable as $H(t)$ (running maximum) which evolves as follows: for $W(t)<H(t), d H(t)=W(t) \eta\left(\frac{d F(t)}{F(t)}-\frac{d Y(t)}{Y(t)}\right)$; for $W(t) \geq H(t), H(t)=W(t)$. This law of motion mimics the evolution of the running maximum of the performance of the fund: an increase of the AUM due to the inflow of funds also increases the running maximum and therefore it is neutral in terms of incentives.

The fund manager maximizes the expected net value of future fees that are paid in continuous time. The management fee is represented by a fraction $a$ of $W(t)$, the HWM component is due when $W(t) \geq H(t)$ and is provided by a constant $k$ multiplied for the increase in the running maximum depurated for the instantaneous in/outflow of funds. We assume that the remuneration is provided by a convex combination of the two components with weight $x \in[0,1]$ for the HWM fee. In this framework, the AUM evolves as

$$
\begin{aligned}
& d W(t)=W(t)[(1-\theta(t)) r+\theta(t) \mu] d t+W(t) \sigma \theta(t) d Z(t) \\
& +W(t) \eta\left(\frac{d F(t)}{F(t)}-\frac{d Y(t)}{Y(t)}\right)-(1-x) a W(t) d t \\
& -x k\left(d H(t)-W(t) \eta\left(\frac{d F(t)}{F(t)}-\frac{d Y(t)}{Y(t)}\right)\right) .
\end{aligned}
$$

As in $[6,9,11]$, we assume that the fund can be liquidated exogenously or endogenously. We denote by $\tau$ the termination time of the fund. The exogenous termination is fully described by a Poisson process of parameter $\lambda>0$. The fund will be terminated endogenously the first time that the AUM touch the liquidation threshold which is defined as a fraction of $\frac{W}{H}$, i.e. the fund is liquidated if $W(t)$ falls to a fraction $b$ of $H(t)$. More precisely, we set $\tau=\min \left\{\tau^{0}, \tau^{1}\right\}$, where we denote by $\tau^{0}$ and by $\tau^{1}$ the stopping times for the exogenous and the endogenous termination, respectively, i.e. $\tau^{0}$ is the first time that the Poisson process jumps and $\tau^{1}=\inf \{t: W(t) \leq b H(t)\}$. We also assume that the manager can't reinvest the fees in a private fund and discounts future revenues at the rate $\gamma>0$. The manager's objective function is of the form

$$
\begin{aligned}
& V(W(0), H(0))=\max _{\theta} \mathbb{E}\left[\int_{0}^{\tau} e^{-\gamma t}\{(1-x) a W(t) d t\right. \\
& \left.\left.+x k\left(d H(t)-W(t) \eta\left(\frac{d F(t)}{F(t)}-\frac{d Y(t)}{Y(t)}\right)\right)\right\}+e^{-\gamma \tau} \underline{V}(\tau)\right] .
\end{aligned}
$$

Upon endogenous/exogenous termination, the manager receives an outside option, i.e. a fraction of the HWM [6]:

$$
\underline{V}(\tau)=c H(\tau), \quad 0 \leq c \leq b
$$

Since $W(t) \geq b H(t)$, the value of the above payoff is smaller (or equal) than the AUM at termination $W(\tau)$.

Let us now consider the AUM HWM contract. In this case, the definition of $H(t)$ is $H(t)=\max _{s}<t W(s)$ and therefore, if $W(t)<H(t)$, then $d H(t)=0$. The objective function becomes

$$
\begin{aligned}
& V(W(0), H(0))=\max _{\theta} \mathbb{E}\left[\int_{0}^{\tau} e^{-\gamma t}\{(1-x) a W(t) d t\right. \\
& \left.+x k d H(t)\}+e^{-\gamma \tau} \underline{V}(\tau)\right],
\end{aligned}
$$

and the AUM evolve as

$$
\begin{aligned}
& d W(t)=W(t)[(1-\theta(t)) r+\theta(t) \mu] d t+W(t) \sigma \theta(t) d Z(t) \\
& +W(t) \eta\left(\frac{d F(t)}{F(t)}-\frac{d Y(t)}{Y(t)}\right)-(1-x) a W(t) d t-x k d H(t)
\end{aligned}
$$

\section{The value function}

We start analyzing the pure performance running maximum HWM contract. We study the behavior of the value function in the region $\{W(t) \geq H(t)\}$. Note that in this region $H(t)=W(t)$. Using the continuity of the value function before and after the adjustment of the HWM, following [9, Appendix A], we obtain

$$
x k V_{W}(H(t), H(t))=x k+V_{H}(H(t), H(t)) .
$$

Then we deal with the region $\{W(t)<H(t)\}$, where $d H(t)=W(t) \eta\left(\frac{d F(t)}{F(t)}-\frac{d Y(t)}{Y(t)}\right)$. In this case the following HJB equation for $V=V(W, H)$ holds true: for any $H>$ $0, W \in(b H, H)$

$$
\begin{aligned}
& 0=-(\gamma+\lambda) V+\lambda \underline{\underline{V}}+\max _{\theta}\left\{V_{W} W[r-(1-x) a\right. \\
& +(1+\eta)(\mu-r) \theta-\eta \beta(\mu-r)]+V_{H} W \eta(\mu-r)(\theta-\beta) \\
& +\frac{1}{2} V_{W W} \sigma^{2} W^{2}[\theta(1+\eta)-\eta \beta]^{2}+\frac{1}{2} V_{H H} \sigma^{2} W^{2} \eta^{2}(\theta-\beta)^{2} \\
& \left.+V_{W H} \sigma^{2} W^{2} \eta(\theta-\beta)[\theta(1+\eta)-\eta \beta]\right\}+(1-x) a W
\end{aligned}
$$


with boundary conditions given by (1)-(2), i.e.

$$
V(b H, H)=\underline{V}=c H, \quad V_{W}(H, H)=1+\frac{V_{H}(H, H)}{x k} .
$$

We would like to stress that the optimization problem is time-homogeneous due to the memory-less property of the exponential arrival time of exogenous liquidation.

A similarity reduction can be applied to simplify the problem. In fact, as in [9], we can exploit an homogeneity property to simplify (3). More precisely, assuming $V(W, H)=H f(w)$, with $w=\frac{W}{H}$, equation (3) becomes

$$
\begin{aligned}
& 0=-(\gamma+\lambda) f+\lambda c+(1-x) a w+\max _{\theta}\left\{w f^{\prime}[r-(1-x) a\right. \\
& +(1+\eta)(\mu-r) \theta-\eta \beta(\mu-r)]+\eta(\mu-r)(\theta-\beta) w\left(f-w f^{\prime}\right) \\
& \left.+\frac{1}{2} \sigma^{2} w^{2} f^{\prime \prime}(\theta(1+\eta-w \eta)-\eta \beta(1-w))^{2}\right\}
\end{aligned}
$$

$\forall w \in(b, 1)$, with boundary conditions $f(b)=c$, and $(1+x k) f^{\prime}(1)-f(1)=x k$. Assuming that there exists a solution to the maximization problem with the right concavity, i.e. $f^{\prime \prime}<0$, we derive the optimal strategy

$$
\begin{aligned}
\theta_{P}^{*} & =\frac{(r-\mu)\left(\eta w f(w)+w f^{\prime}(w)(1+\eta-w \eta)\right)}{\sigma^{2} w^{2} f^{\prime \prime}(w)(1+\eta-w \eta)^{2}} \\
& +\frac{\eta \beta(1-w)}{1+\eta-w \eta} .
\end{aligned}
$$

An analytical solution of this ordinary differential equation is not available. Therefore, we use an iterative numerical algorithm, based on a finite difference scheme, to compute the optimal strategy $\theta_{P}^{*}$ and the value function $V$. We refer to [1] for details. A verification theorem can be proved following [11, Appendix A].

Considering the assets under management HWM contract and recalling that $d H(t)=0$ in the region $W(t)<$ $H(t)$, equation (4) becomes: $\forall w \in(b, 1)$

$$
\begin{aligned}
& 0=-(\gamma+\lambda) f+\lambda c+(1-x) a w+\max _{\theta}\left\{w f^{\prime}[r-(1-x) a\right. \\
& \left.+(1+\eta)(\mu-r) \theta-\eta \beta(\mu-r)]+\frac{1}{2} \sigma^{2} w^{2} f^{\prime \prime}[\theta(1+\eta)-\eta \beta]^{2}\right\}
\end{aligned}
$$

and, assuming that $f^{\prime \prime}<0$, the optimal strategy is

$$
\theta_{A U M}^{*}=\frac{1}{1+\eta}\left(\eta \beta-\frac{f^{\prime}(w)}{w f^{\prime \prime}(w)} \frac{\mu-r}{\sigma^{2}}\right) .
$$

\section{The optimal investment strategy}

First of all, we analyze the optimal investment strategy associated with the two incentive fee remuneration schemes assuming a liquidation threshold at $0(b=0)$ and a strictly positive liquidation threshold $(b>0)$. In Figure 1 we plot the optimal strategies $\theta_{P}^{*}, \theta_{A U M}^{*}$ defined in (5) and (6) as a function of $w$. Note that the two strategies coincide for $\eta=0$. As a reference, we also plot the optimal investment strategy defined in [11], i.e. $\theta_{P W}^{*}=\frac{\mu-r}{(1-\alpha) \sigma^{2}}$ with

$$
\alpha=\frac{\gamma+\lambda+r+\pi-\sqrt{(\gamma+\lambda+r+\pi)^{2}-4 r(\gamma+\lambda)}}{2 r},
$$

and $\pi=\frac{1}{2}\left(\frac{\mu-r}{\sigma}\right)^{2}$, which is optimal in case of no liquidation threshold $(b=0)$, no management fee $(a=0)$ and no flow of funds $(\eta=0)$.

The strategy presented in [11] is of a constant weight type, but it is characterized by a significant risk exposure. Instead, the strategies associated with our two incentive fee remuneration schemes are not constant, but depends on the fraction $w=W / H$.

We observe that the optimal strategy depends on the definition of the HWM contract. A contract with a maximum defined on the AUM $\left(\theta_{A U M}^{*}\right)$ leads to a strategy that is less aggressive than the one obtained in case of a contract defined as a pure running maximum on the performance of the fund $\left(\theta_{P}^{*}\right)$. The rationale of this result goes back to the trade-off between downside liquidation risk and the benefit from continuation: in case of a pure running maximum the flow of funds doesn't affect the maximum and therefore the future remuneration from incentive fees is less risky than in case the flow of funds is included. Therefore, the manager takes a more aggressive strategy with a pure running maximum incentive fee than in case of a remuneration scheme where the maximum depends on the in/outflow of funds.

The optimal strategy depends upon the endogenous liquidation trigger. Comparing the two plots of Figure 1 we observe that manager invests in the risky asset more in the case of liquidation trigger posed at zero $(b=0)$ than in the case of a strictly positive liquidation trigger $(b>0)$. The rationale is that going from $b=0$ to $b>0$ the downside liquidation risk increases leading to a smaller risk exposure. The increasing shape in case of a strictly positive liquidation trigger can be explained through the trade-off between the liquidation risk and the remuneration associated with a continuation: next to the threshold the liquidation risk is high and therefore the manager reduces its risk exposure.

We now turn to a comparative statics of the optimal investment strategies. We limit our analysis to the case $b>0, c>0$. In Figure 2 we compare the optimal strategies $\theta_{P}^{*}$ defined in (5), and $\theta_{A U M}^{*}$ defined in (6) with the benchmark's strategy $\beta$ and the HWM optimal investment strategy obtained in case of no flow of funds $(\eta=0)$.

First of all we provide a comparative statics analysis with respect to the parameters $x, b, c$, Figure 2.(a), (b), (c), respectively. Increasing the weight of the HWM fee with respect to the management one (increasing $x$ ), the asset manager adopts a more aggressive optimal investment strategy. This result can be rationalized observing that the management fee component of the contract is linear on the AUM, instead the HWM incentive fee is an option like component. As a consequence, when the latter component becomes more important with respect to the 

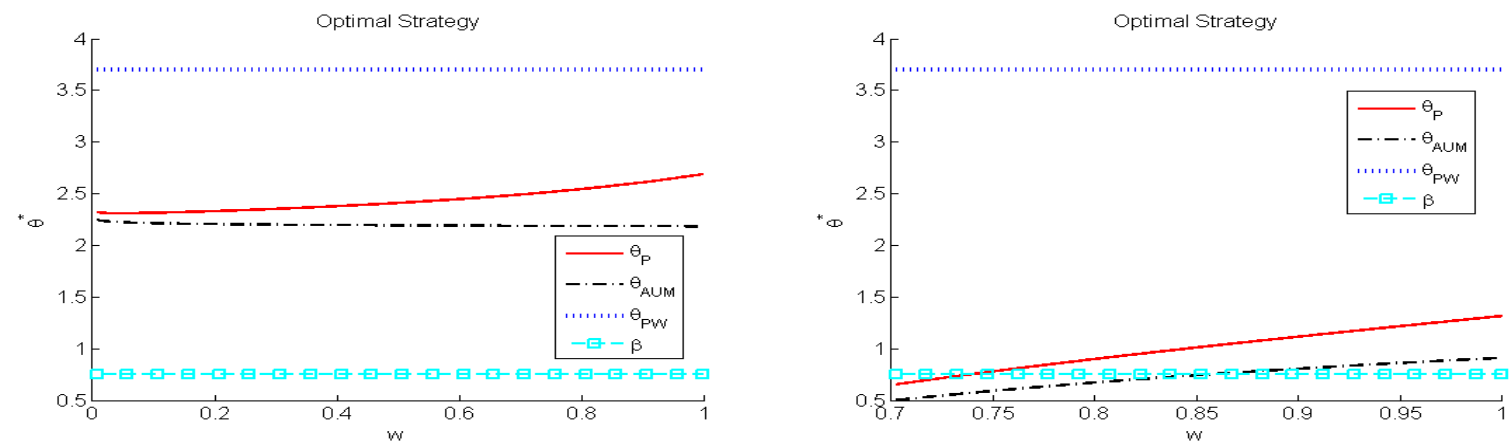

Figure 1: Optimal strategy $\theta^{*}$. Parameters: $r=0.02, \mu=0.06, \sigma=0.5, \gamma=0.04, \lambda=0.05, \eta=1.2, \beta=0.75, k=0.4, a=0.04, x=0.5$, $b=c=0$ (left) $b=0.7, c=0.4$ (right).

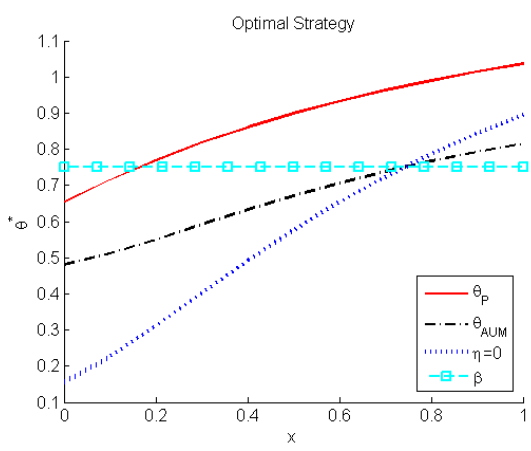

(a) $\theta^{*}$ varying $x$

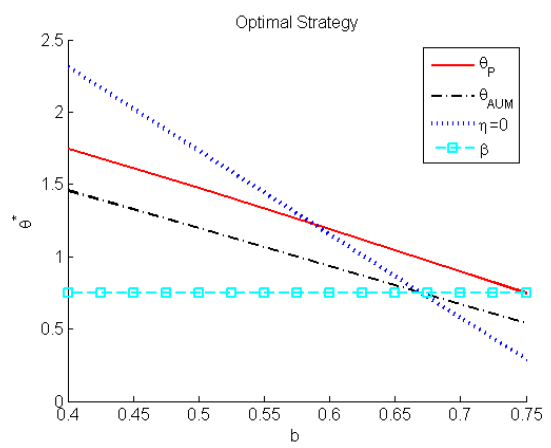

(b) $\theta^{*}$ varying $b$

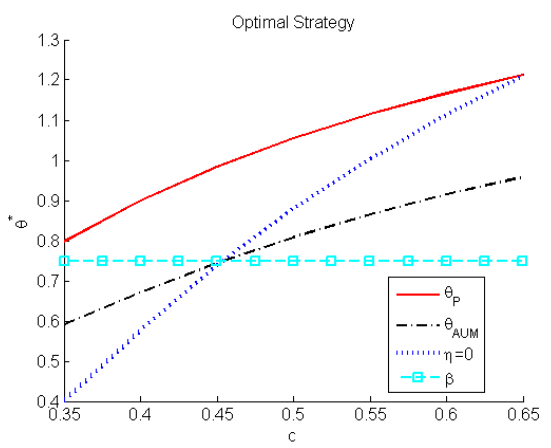

(c) $\theta^{*}$ varying $c$

Figure 2: Optimal strategy $\theta^{*}$ in $w=0.8$. Base parameters: $r=0.02, \mu=0.06, \sigma=0.5, \gamma=0.04, \lambda=0.05, \eta=1.2, \beta=0.75, k=0.4$, $a=0.04, x=0.5, b=0.7, c=0.4$

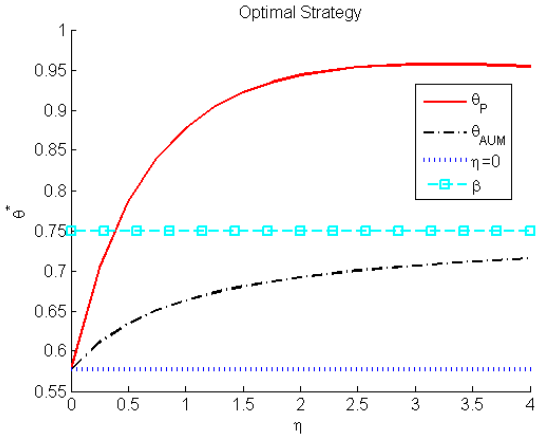

(a) $\theta^{*}$ varying $\eta$

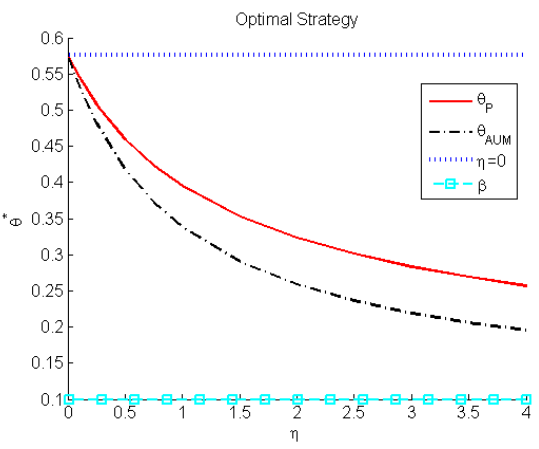

(b) $\theta^{*}$ varying $\eta$

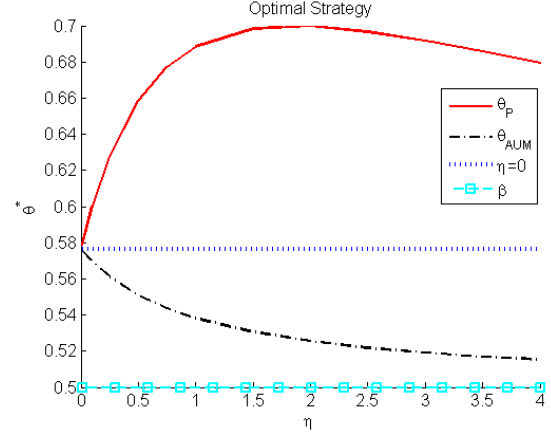

(c) $\theta^{*}$ varying $\eta$

Figure 3: Optimal strategy $\theta^{*}$ in $w=0.8$. Base parameters: $r=0.02, \mu=0.06, \sigma=0.5, \gamma=0.04, \lambda=0.05, \beta=0.75($ left $) \beta=0.1$ (center) $\beta=0.5$ (right), $k=0.4, a=0.04, x=0.5, b=0.7, c=0.4$

first one the asset manager takes more risk. As far as $b$ and $c$ is concerned, we observe that as $b$ decreases, the downside risk decreases and therefore the investment in the risky asset increases. The opposite effect is observed for $c$ that determines the scrap value in case of endogenous/exogenous termination: when the termination value increases, the manager increases the investment as the possibility of an endogenous termination is not so unpleasant.

Figures 3 and 4 describe the role of $\eta$ on the optimal strategies for different values of the benchmark $\beta$. The first figure shows that the optimal investment strategy in case of a HWM defined on the AUM $\left(\theta_{A U M}^{*}\right)$ tends to the benchmark as the flow fund effect increases. This convergence effect does not hold on $\theta_{P}^{*}$. Figure 3 also shows the convergence of the optimal strategies to the case without flow of funds when $\eta \rightarrow 0$ : the convergence can be from above or below, and can also be non monotonic in case of $\theta_{P}^{*}$, depending on the value of the benchmark. Figure 4 confirm the convergence to the benchmark for the $\theta_{A U M}^{*}$ strategy. Figure 4 provides the optimal strategies varying 


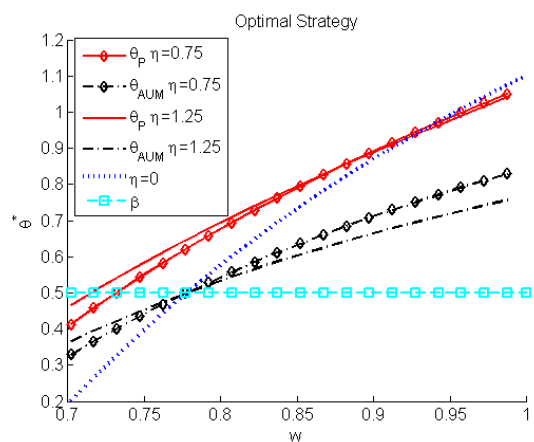

(a)

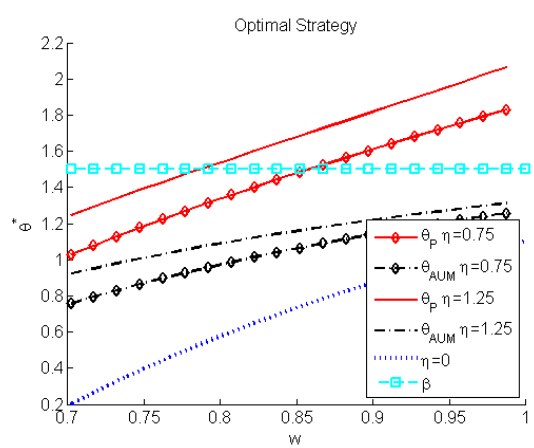

(b)

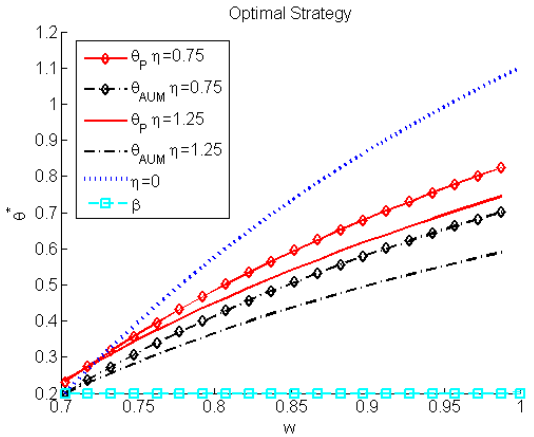

(c)

Figure 4: Optimal strategy $\theta^{*}$. Base parameters: $r=0.02, \mu=0.06, \sigma=0.5, \gamma=0.04, \lambda=0.05, \beta=0.5$ (left) $\beta=1.5$ (center) $\beta=0.2$ (right), $k=0.4, a=0.04, x=0.5, b=0.7, c=0.4$.

the benchmark and the optimal strategy for $\eta=0$ : in the first case the optimal strategy for $\eta=0$ intercepts the benchmark, in the second case it lies below the benchmark for all values of $w$, in the third case it is always above.

It turns out that the strategy $\theta_{A U M}^{*}$ always lies between the benchmark and the optimal investment strategy for $\eta=0$. As $\eta$ increases, the benchmark role increases and $\theta_{A U M}^{*}$ tends to $\beta$. So we can conclude that thanks to the flow of funds a HWM defined on the AUM induces the asset manager to take a less extreme investment strategy. On the opposite, the strategy $\theta_{P}^{*}$ is characterized by a higher risk exposure than that of the optimal investment strategy for $\eta=0$ unless in case of a low $b$ or $\beta$. In the case of higher (lower) risk exposure the phenomenon is amplified (dampened) as $\eta$ increases.

These results show the potential negative effect that comes from a combination between a non well designed HWM contract and the flow of funds. We may assume that an investor looks for an aggressive strategy by a manager of a hedge fund but we may consider as "expected" risk exposure the risk taking level obtained without flow of funds, and "unwanted" the extra risk taking exposure that comes from the flow of funds.

\section{References}

[1] Barucci, Emilio and Marazzina, Daniele (2015) Risk seeking, nonconvex remuneration and regime switching, International Journal of Theoretical and Applied Finance, 18(2): 1550009.

[2] Basak, Suleyman, Pavlova, Anna and Shapiro, Alexander (2007) Optimal asset allocation and risk shifting in money management, Review of Financial Studies, 20: 1583-1621.

[3] Browne, Sid (1995) Optimal investment policies for a firm with a random risk process: exponential utility and minimizing the probability of ruin, Mathematics of Operations Research, 20(4): 937-957.

[4] Carpenter, Jennifer (2000) Does option compensation increase managerial risk appetite?, Journal of Finance, LV: 2311-2331.

[5] Chevalier, Judith and Ellison, Gleen (1997) Risk taking by mutual funds as a response to incentives, Journal of Political Economy, 105: 1167-1200.

[6] Drechsler, Itamar (2014) Risk choice under high-water marks, Review of Financial Studies, 27: 2052-2096.
[7] Goetzmann, Wiliam, Ingersoll, Jonathan and Ross, Stephen (2003) High water marks and hedge fund management contracts, Journal of Finance, 58: 1685-1717.

[8] Grinblatt, Mark and Titman, Sheridan (1989) Adverse risk incentives and the design of performance-based contracts, Management Science, 35: 807-822.

[9] Lan, Yincong, Wang, Neng and Yang, Jinqiang (2013) The economics of hedge funds, Journal of Financial Economics, 110(2): 300-323.

[10] Merton Robert (1971) Optimum consumption and portfolio rules in a continuous-time model, Journal of Economic Theory, 3(4): 373-413.

[11] Panageas, Stavros and Westerfield, Mark (2009) High-Water Marks: High risk appetites? Convex compensation, long horizons, and portfolio choice, Journal of Finance, 64(1): 1-36.

[12] Sirri, Robert and Tufano, Peter (1998) Costly search and mutual fund flows, Journal of Finance, 54: 359-375. 